\title{
Corrective Septorhinoplasty in Acute Nasal Bone Fractures
}

\author{
Jisung Kim · Hahn Jin Jung · Woo Sub Shim \\ Department of Otorhinolaryngology-Head and Neck Surgery, Chungbuk National University Hospital, Chungbuk National University College of \\ Medicine, Cheongju, Korea
}

Objectives. Closed reduction is generally recommended for acute nasal bone fractures, and rhinoplasty is considered in cases with an unsatisfactory outcome. However, concomitant rhinoplasty with fracture reduction might achieve better surgical outcomes. This study investigated the surgical techniques and outcomes in patients who underwent rhinoplasty and fracture reduction concomitantly, during the acute stage of nasal bone fracture.

Methods. Forty-five patients who underwent concomitant rhinoplasty and fracture reduction were enrolled. Nasal bone fractures were classified into three major types (type I, simple fracture; type II, fracture line that mimics nasal osteotomy; and type III, comminuted fracture) based on computed tomography images and preoperative facial images. Two independent otolaryngology-head and neck surgeons evaluated the surgical outcomes and telephone based survey were made to evaluate patients satisfaction.

Results. Among 45 patients, there were 39 males and 6 females. Type I was the commonest type of fracture with 18 patients $(40 \%)$, while the most frequently used surgical technique for corrective surgery was dorsal augmentation with 44 patients $(97.8 \%)$. The mean visual analogue scale satisfaction score of the surgeons and patients were 7.62 and 8 , respectively, with no significant differences between fracture types.

Conclusion. Concomitant rhinoplasty with fracture reduction can be performed for acute nasal bone fracture patients, and it might lead to better aesthetic outcomes.

Keywords. Nasal Bone; Bone Fractures; Rhinoplasty

\section{INTRODUCTION}

The nose is the most protuberant feature of the face, and thus, most vulnerable to facial trauma [1]. Hence, nasal bone fracture is reported to be the most common facial fracture and the third most common fracture in the human body [2].

The techniques for treating a nasal bone fracture are closed reduction or open reduction; closed reduction within 3 weeks of injury is usually recommended [3-5]. This is also because closed

\footnotetext{
- Received March 30, 2017

Revised May 16, 2017

Accepted May 25, 2017

- Corresponding author: Woo Sub Shim

Department of Otorhinolaryngology-Head and Neck Surgery, Chungbuk National University Hospital, Chungbuk National University College of

Medicine, 776 1sunhwan-ro, Soewon-gu, Cheongju 28644, Korea

Tel: +82-43-269-6157, Fax: +82-43-265-6157

E-mail: hwatas@naver.com
}

reduction is a relatively simple procedure that can be performed on an outpatient-basis, under local anesthesia; thus it is cost-effective [6,7]. However, outcomes following closed reduction are difficult to predict and reports of postreduction external nasal deformity vary from $9 \%$ to $50 \%$ [2,6,8-11]. Additionally, some cases require open reduction with rhinoplasty in the early stage $[10,12,13]$.

Even a simple fracture can lead to a nasal deformity, and inappropriate treatment can cause aesthetic and functional issues. External nasal deformity prior to nasal trauma may lead to unexpected outcomes. Hence, initial treatment needs to be planned carefully. In the present study, we aimed to analyze the clinical features in cases where concomitant rhinoplasty and open reduction were performed as the primary treatment, after nasal bone fracture. Furthermore, we concentrated on patients who had external nasal deformity prior to nasal bone fracture.

Copyright (C) 2018 by Korean Society of Otorhinolaryngology-Head and Neck Surgery.

This is an open-access article distributed under the terms of the Creative Commons Attribution Non-Commercial License (http://creativecommons.org/licenses/by-nc/4.0)

which permits unrestricted non-commercial use, distribution, and reproduction in any medium, provided the original work is properly cited. 
Table 1. Nasal bone fracture classification

\begin{tabular}{ll}
\hline Classification & \multicolumn{1}{c}{ Description } \\
\hline II & Simple fracture with minimal displacement \\
IIb & Fracture with a favorable fracture line that mimics nasal osteotomy, which is performed for improving the shape of the nose \\
III & A broad nasal dorsum on the unaffected side \\
\hline
\end{tabular}
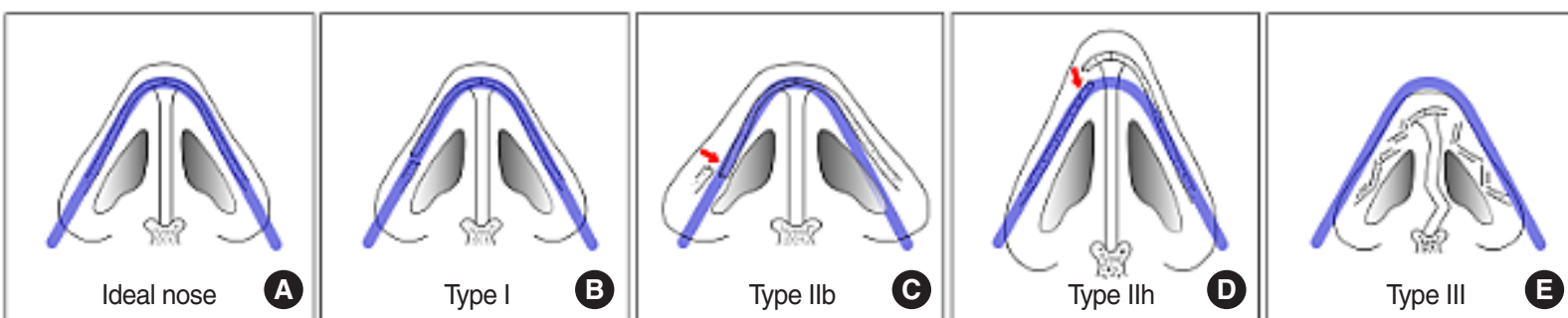

Fig. 1. Types of nasal bone fractures. Blue line indicates an ideal shape and location of the nasal bone. (A) Aesthetically ideal bony pyramid. (B) Type I, minimally displaced nasal bone fracture, which remains within the blue line. (C) Type Ilb, a broad based nose, with unaffected bone outside the blue line and the fractured bone inside the blue line. (D) Type Ilh, a hump nose with intact bone above the blue line and fractured bone inside the blue line. (E) Type III, comminuted fracture with a septal fracture where all components collapse away from the blue line. Arrows indicate directions of displacement of fracture site in favorable way.

\section{MATERIALS AND METHODS}

The present study was a retrospective analysis of pre- and postoperative facial photographs and computed tomography (CT) images, as well as medical records of 45 patients who underwent concomitant rhinoplasty and open reduction within 4 weeks of nasal bone fracture. Regardless of fracture type, patients' personal desire for an aesthetic improvement was our major guideline to choose which patient does or does not receive rhinoplasty. This study was approved by Institutional Review Board of Chungbuk National University Hospital (IRB No. 2016-05-010).

The procedures were performed at the Department of Otorhinolaryngology in Chungbuk National University Hospital between January 2010 and June 2016. The inclusion criteria were patients whose outpatient follow-up records were available for at least 6 months following the surgical treatment. Patients with a history of rhinoplasty, nasal septoplasty, or nasal bone fracture prior to the most recent injury were excluded from the study. The mean follow-up period was 3.25 years (range, 6 months to 6 years). Age, sex, mean time between trauma and surgery, and type of surgery patients received were documented based on

\section{H I G HLI I G H T S}

- Closed reduction in nasal bone fracture is relatively simple procedure.

- The outcomes of closed reduction are difficult to predict.

- Concomitant rhinoplasty and open reduction might achieve better surgical outcome in selected cases. medical records. Nasal bone fracture was classified by a novel method made by the investigators into the following types based on pre- and posttrauma photographs and CT images of the patient (Table 1, Fig. 1).

Two otorhinolaryngologists, who did not participate in any surgeries for these patients, assessed the surgical outcomes. The assessment involved an analysis of pre- and postoperative facial photographs and CT images of the patients, and use of a visual analog scale (VAS) for assessor's satisfaction, where a score of 0 indicated "not satisfied at all" and 10 indicated "very satisfied." Same VAS method was used for patient's satisfaction that were obtained by telephone based survey.

The IBM SPSS ver. 21.0 (IBM Corp., Armonk, NY, USA) was used for statistical analysis. A chi-square test was performed for the analysis of fracture types and surgical methods used. To determine the correlations of surgical methods with VAS scores, a student $t$-test was performed.

\section{RESULTS}

Among 45 patients, there were 39 males (86.7\%) and 6 females (13.3\%), with a mean age of 29.3 years (range, 15 to 65 years). The mean time between the nasal bone fracture and surgery was 17.8 days (range, 6 to 28 days). Type I was the commonest type of fracture with 18 patients $(40 \%)$, while the most frequently used surgical technique for corrective surgery was dorsal augmentation with 44 patients (97.8\%) (Table 2).

The types of corrective surgeries performed were divided into three groups and the outcomes were compared between the 
groups. Spreader graft was the most commonly used technique in type II fractures $(n=9,81.8 \%)$ and extracorporeal septoplasty was most commonly used in type III fractures $(n=12,75 \%)$. The

Table 2. Characteristics of patients who received rhinoplasty after nasal bone fracture

\begin{tabular}{lr}
\hline Characteristic & No. (\%) \\
\hline Sex & $39(86.7)$ \\
Male & $6(13.3)$ \\
\hline Female & \\
Nasal bone fracture type & $18(40.0)$ \\
\hline I & $6(13.3)$ \\
IIb & $5(11.1)$ \\
III & $16(35.6)$ \\
Surgical technique & \\
Dorsal augmentation & \\
\hline Implant & $35(77.8)$ \\
Onlay graft & $33(73.3)$ \\
Septal extension graft & $13(28.9)$ \\
Dorsal reinforcement & \\
Extracorporeal septoplasty & $18(40.0)$ \\
Spreader graft & $14(31.1)$ \\
\hline Tip surgery & \\
Cap graft & $28(62.2)$ \\
Columellar strut & $28(62.2)$ \\
Transdomal suture & $25(55.6)$ \\
Shield graft & $15(33.3)$ \\
Cephalic resection & $1(2.2)$ \\
Osteotomy & $11(14.4)$ \\
\hline
\end{tabular}

mean postoperative VAS score was $7.62 \pm 1.11$ and $8 \pm 0.74$ for surgeon's and patients' respectively, with no significant differences between fracture types (Table 3). When type II fractures were analyzed by sub-types, IIb and IIh, the results show no significant differences in the surgical techniques used orVAS scores (Table 4).

Two cases $(4.4 \%)$ required a revision surgery using auricular cartilage under local anesthesia: one to correct mild asymmetric nasal dorsum and the other to correct mild nasal tip asymmetry. Besides the two cases that we mentioned, there were no reported cases of complications, such as infection, bleeding, nasal septum perforation, or major external nasal deformity in any of the 45 cases. Here we demonstrate two cases of type II fracture (type IIb and type IIh).

\section{Case 1}

A 34-year-old male patient was admitted for a deviated nose and nasal stuffiness caused by a nasal bone fracture sustained 1 week earlier. The patient had been always unhappy about his broad nasal dorsum and it was explained to him that the fracture line caused by the trauma mimicked nasal osteotomy for correcting a broad nasal dorsum. Based on CT images and shape of the patient's nose, it was diagnosed as a type IIb nasal bone fracture. The surgery involved medial and lateral nasal osteotomy using an open external rhinoplasty approach on the unaffected side. Augmentation surgery was performed using silicone, and cap graft was performed by raising a columellar strut and spreader graft with the nasal septal cartilage. The patient had a natural looking nasal bridge after the surgery, and nasal stuffi-

Table 3. Analysis of rhinoplasty procedures and surgical outcomes

\begin{tabular}{|c|c|c|c|c|c|c|c|c|c|}
\hline \multirow{3}{*}{ Fracture type } & \multicolumn{7}{|c|}{ Rhinoplasty method } & \multicolumn{2}{|c|}{ VAS } \\
\hline & \multicolumn{3}{|c|}{ Dorsal augmentation } & \multicolumn{2}{|c|}{ Dorsal reinforcement } & \multirow{2}{*}{ Tip surgery } & \multirow{2}{*}{ Osteotomy } & \multirow{2}{*}{ Surgeon } & \multirow{2}{*}{ Patient } \\
\hline & Implant & Onlay & SEG & Spreader & ECS & & & & \\
\hline I & $14(77.8)$ & $12(66.7)$ & $6(33.3)$ & $2(11.1)$ & $4(22.2)$ & $18(100)$ & 0 & $7.72 \pm 1.18$ & $8.11 \pm 0.76$ \\
\hline II & $9(81.8)$ & $8(72.7)$ & $4(36.4)$ & $9(81.8)^{\star}$ & $2(18.2)$ & $11(100)$ & $11(100)^{*}$ & $7.36 \pm 1.50$ & $7.82 \pm 0.75$ \\
\hline III & $12(75)$ & $13(81.3)$ & $3(18.8)$ & $3(18.8)$ & $12(75)^{\star}$ & $12(75)$ & 0 & $7.69 \pm 0.70$ & $8 \pm 0.73$ \\
\hline Total & - & - & - & - & - & - & - & $7.62 \pm 1.11$ & $8 \pm 0.74$ \\
\hline$P$-value & 0.916 & 0.630 & 0.529 & $<0.001$ & 0.002 & 0.972 & $<0.001$ & 0.683 & 0.627 \\
\hline
\end{tabular}

Values are presented as number (\%) or mean \pm standard deviation.

VAS, visual analogue scale; Onlay, onlay graft; SEG, septal extension graft; Spreader, spreader graft; ECS, extracorporeal septoplasty.

*Statistically significant $(P<0.05)$.

Table 4. Analysis of rhinoplasty procedures and surgical outcomes within type II fractures

\begin{tabular}{|c|c|c|c|c|c|c|c|c|c|}
\hline \multirow{3}{*}{ Fracture type } & \multicolumn{7}{|c|}{ Rhinoplasty method } & \multicolumn{2}{|c|}{ VAS } \\
\hline & \multicolumn{3}{|c|}{ Dorsal augmentation } & \multicolumn{2}{|c|}{ Dorsal reinforcement } & \multirow{2}{*}{ Tip surgery } & \multirow{2}{*}{ Osteotomy } & \multirow{2}{*}{ Surgeon } & \multirow{2}{*}{ Patient } \\
\hline & Implant & Onlay & SEG & Spreader & ECS & & & & \\
\hline $\mathrm{Ilb}$ & $6(100)$ & $4(66.7)$ & $1(16.7)$ & $5(83.3)$ & $1(16.7)$ & $6(100)$ & $6(100)$ & $7.83 \pm 0.98$ & $8 \pm 0.63$ \\
\hline Ilh & $3(60)$ & $4(80)$ & $3(60)$ & $4(80)$ & $1(20)$ & $5(100)$ & $5(100)$ & $6.80 \pm 1.92$ & $7.6 \pm 0.89$ \\
\hline$P$-value & 0.182 & 0.576 & 0.197 & 0.727 & 0.727 & 1 & 1 & 0.278 & 0.284 \\
\hline
\end{tabular}

Values are presented as number (\%) or mean \pm standard deviation.

VAS, visual analogue scale; Onlay, onlay graft; SEG, septal extension graft; Spreader, spreader graft; ECS, extracorporeal septoplasty. 

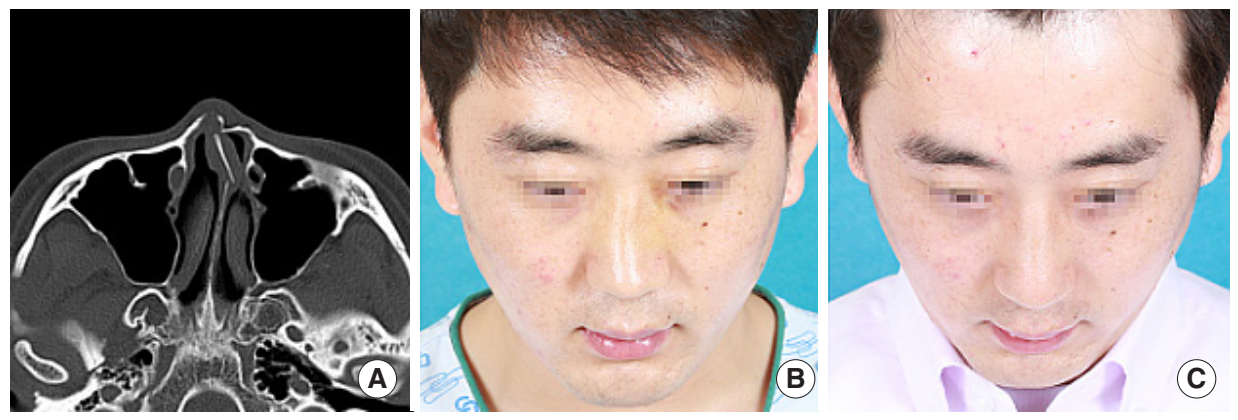

Fig. 2. Facial photographs and computed tomography (CT) image of case 1. (A) Preoperative facial bone CT shows a broad base bony pyramid with left nasal bone fracture. The fracture line mimics a lateral osteotomy. (B) Preoperative facial photograph shows a right side broad base nose. (C) Postoperative facial photograph shows a well-corrected broad base nose.
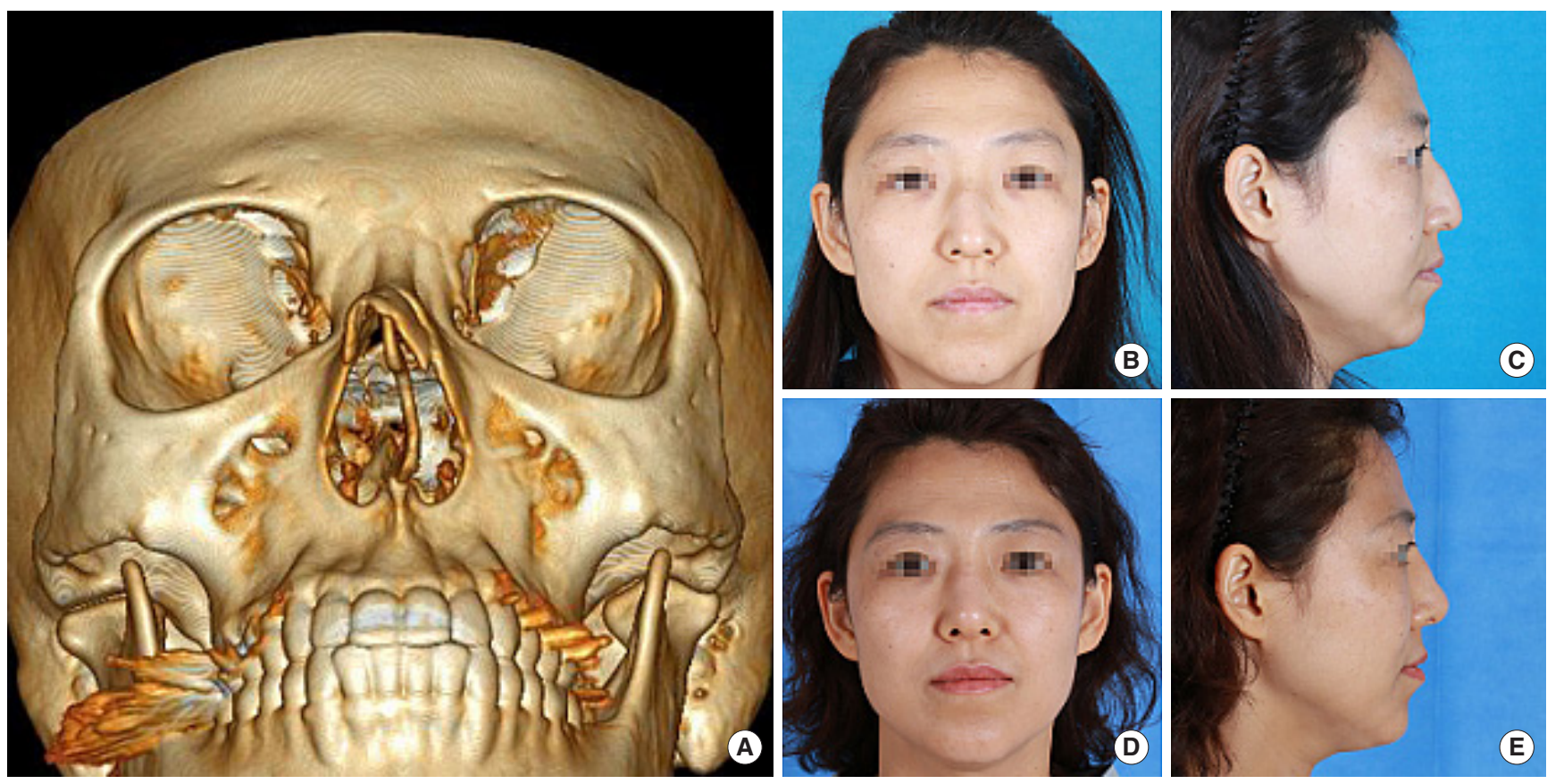

Fig. 3. Facial photographs and three-dimensionally reconstructed computed tomography (CT) image of case 2. (A) Preoperative three-dimensionally reconstructed CT image shows a hump nose with left nasal bone fracture. The fracture lines mimic a medial and lateral osteotomies. $(B, C)$ Preoperative facial photographs show a hump nose with right side deviation. (D, E) Postoperative facial photograph shows a well-corrected hump nose.

ness improved, resulting in high satisfaction level in both, the patient and surgeon (Fig. 2).

\section{Case 2}

A 29-year-old female patient was admitted for nasal bone and septal fractures sustained 2 weeks earlier. The patient had been always unhappy about her hump nose. Her fracture was classified as type IIh nasal bone fracture based on CT images and shape of the patient's nose. It was explained to the patient that the pattern of her fracture mimicked nasal osteotomy, which is performed to correct an open roof deformity that occurs following corrective surgery on hump nose. The patient agreed to undergo external rhinoplasty and open reduction. Since the patient desired a natural looking long nose, the surgery involved reduction of nasal bone fracture via an open external rhinoplasty approach, followed by medial and lateral nasal osteotomy and nasal hump resection for correction of the hump nose, in addition to nasal augmentation using silicone. The patient had a natural looking nasal dorsum after the surgery and the satisfaction level was high in both, the patient and the surgeon (Fig. 3).

\section{DISCUSSION}

In the present study, nasal bone fractures were classified into three types: type I was a simple fracture, for which concomitant immediate reduction and rhinoplasty could be performed if the patient desired cosmetic rhinoplasty. Closed reduction is often used as the primary treatment for simple nasal bone fractures, and if the treatment fails, open reduction or rhinoplasty is considered after reevaluation at 6 months $[6,14]$. On the other hand, a recent study successfully performed immediate reduc- 
tion and rhinoplasty after a nasal bone fracture and indicated a surgeons' mean VAS score of 7.14, which was similar to the results of our present study [13]. An open approach allows more accurate assessment and reduction of the fractured nasal bone and septum. Moreover, the patient's desire for cosmetic rhinoplasty can be fulfilled through a diligent surgery, which also reduces the time and cost associated with secondary surgery.

Type II was defined in the present study as a favorable fracture, which is similar to an osteotomy performed during rhinoplasty. In rhinoplasty, osteotomy is performed to correct the broad base of the nose, deviated nose, or open roof deformity caused by nasal hump resection. When a fracture similar to osteotomy occurs in patients with a naturally flat or hump nose, correction of the nasal bone fracture is performed concomitantly with rhinoplasty, via osteotomy on the unaffected side.

Type III was comminuted fracture, which often requires open reduction and internal fixation. For this, internal fixation with a microplate is done after open sky incision, but the surgical outcome may not be satisfactory due to visible scarring, or the skin above the microplate being prominent or palpable. Although such deformity can be reduced by closed reduction, followed by rhinoplasty as a secondary procedure, the prolonged period of treatment and hospitalization, increases the treatment costs. In general, changes in the rate of bone resorption, adhesion to nearby tissues, and stiffening of the supporting base are expected in the acute phase of a nasal bone fracture; hence, there is reluctance for concomitant fracture reduction and cosmetic surgery. In contrast, there are studies that recommend rhinoplasty using an open approach for elaborate, functional reconstruction of comminuted nasal bone fracture $[5,15]$ while the present study showed successful surgical outcomes by performing augmentation surgery after reconstruction of the caudal septum when the radix was intact.

We found that spreader graft and osteotomy were the most commonly used technique in type II fractures, and extracorporeal septoplasty was most commonly used in type III fracture with the differences being statistically significant. Although these results may have no clinical meaning, considering the fact that choosing which surgical techniques to implement are sometimes decided during septorhinoplasty, data may help explaining to the patients about the procedures that they may receive.

There could be some challenges that should be highlighted in the present study. One is the fact that facial edema and other injuries immediately after the trauma make it difficult to identify the nasal deformity accurately. Nasal deviation may be underestimated due to the presence of facial edema, and height of the nose might be assessed incorrectly due to swelling of the nearby tissues or the presence of a facial fracture. Therefore, when planning rhinoplasty in a patient with nasal bone fracture, thorough preoperative consultation, along with analysis of $\mathrm{CT}$ images and facial photographs of the patient prior to the trauma are essential. Moreover, stability of the radix area must be secured; how- ever, when there is a severe nasal septal fracture, cartilage available for the surgery might be limited, and the use of auricular and/or costal cartilage should be considered.

This was a retrospective study and all patients had undergone rhinoplasty using open approach as the primary treatment. The study has a limitation of not being able to offer a comparative analysis with treatment outcomes from staged rhinoplasty. Hence, additional studies with a larger sample size and various treatment methods are necessary in the future.

A nasal bone fracture can cause aesthetic and functional challenges, and if these challenges are neglected, the patient might require longer hospitalization and treatment period, besides incurring a higher cost of treatment. If the patient desires aesthetic improvement and the outcome of closed reduction is not satisfactory, concomitant open reduction and rhinoplasty can be performed during primary treatment to avoid a secondary surgery, thus optimizing the costs.

In conclusion, for patients with nasal bone fracture with preexisting external nasal deformity, concomitant fracture reduction, and external rhinoplasty through proper selection and consultation with the patient, might lead to better aesthetic outcomes.

\section{CONFLICT OF INTEREST}

No potential conflict of interest relevant to this article was reported.

\section{ACKNOWLEDGMENTS}

This work was supported by a research grant from Chungbuk National University, Cheongju, Republic of Korea, in 2014.

\section{REFERENCES}

1. Rhee SC, Kim YK, Cha JH, Kang SR, Park HS. Septal fracture in simple nasal bone fracture. Plast Reconstr Surg. 2004 Jan;113(1):4552.

2. Murray JA, Maran AG, Mackenzie IJ, Raab G. Open v closed reduction of the fractured nose. Arch Otolaryngol. 1984 Dec;110(12): 797-802.

3. Haug RH, Prather JL. The closed reduction of nasal fractures: an evaluation of two techniques. J Oral Maxillofac Surg. 1991 Dec;49 (12):1288-92.

4. Renner GJ. Management of nasal fractures. Otolaryngol Clin North Am. 1991 Feb;24(1):195-213.

5. Pollock RA. Nasal trauma: pathomechanics and surgical management of acute injuries. Clin Plast Surg. 1992 Jan;19(1):133-47.

6. Mondin V, Rinaldo A, Ferlito A. Management of nasal bone fractures. Am J Otolaryngol. 2005 May-Jun;26(3):181-5.

7. Watson DJ, Parker AJ, Slack RW, Griffiths MV. Local versus general anaesthetic in the management of the fractured nose. Clin OtolaryngolAllied Sci. 1988 Dec;13(6):491-4. 
8. Rohrich RJ, Adams WP Jr. Nasal fracture management: minimizing secondary nasal deformities. Plast Reconstr Surg. 2000 Aug;106(2): 266-73.

9. Murray JA, Maran AG. The treatment of nasal injuries by manipulation. J Laryngol Otol. 1980 Dec;94(12):1405-10.

10. Fernandes SV. Nasal fractures: the taming of the shrewd. Laryngoscope. 2004 Mar;114(3):587-92.

11. Staffel JG. Optimizing treatment of nasal fractures. Laryngoscope. 2002 Oct;112(10):1709-19.

12. Clark WD, Stiernberg CM. Early aggressive treatment of nasal frac- tures. Ear Nose Throat J. 1986 Oct;65(10):481-3.

13. Kim JH, Lee JW, Park CH. Cosmetic rhinoseptoplasty in acute nasal bone fracture. Otolaryngol Head Neck Surg. 2013 Aug;149(2):212-8.

14. Chen CT, Hu TL, Lai JB, Chen YC, Chen YR. Reconstruction of traumatic nasal deformity in Orientals. J Plast Reconstr Aesthet Surg. 2010 Feb;63(2):257-64.

15. Wong $\mathrm{CH}$, Daniel RK. Immediate functional and cosmetic open rhinoplasty following acute nasal fractures: our experience with Asian noses. Aesthet Surg J. 2013 May;33(4):505-15. 